TRANS · núm. $24 \cdot 2020$

DOSIER $\cdot 53-70$

En el presente trabajo se ofrece un análisis estadístico de los cómics traducidos en España a lo largo de los años más álgidos de la crisis económica (2009-2015) con la finalidad de obtener cifras lo más exactas posible sobre el volumen de producción del medio y desvelar el peso de estas en el mercado editorial. En el análisis de estos datos se presta especial atención al impacto que desempeñan las lenguas de los textos de origen en los últimos años en España.

PALABRAS CLAVE: traducción, industria editorial, cómic, novela gráfica, crisis económica.

\title{
La producción editorial de cómics traducidos en la España de la crisis (2009-2015)
}

\section{The Editorial Production of Translated Comics in the \\ Spain of the Crisis (2009-2015)}

ÁNGELO NÉSTORE Universidad de Málaga

This article is aimed at presenting a statistical analysis of translated comics in Spain during the most severe years of the economic crisis (20092015). Its purpose is obtaining as precise figures as possible on the volume of production of this genre and unveiling the trends on the editorial processes from a translation perspective. Special attention is given to the impact of the original languages of the texts in recent years in Spain.

KEY WORDS: translation, publishing industry, comic, graphic novel, economic crisis. 


\section{INTRODUCCIÓN}

En este artículo, tras presentar el estado de la cuestión sobre la realidad de la novela gráfica en España y los problemas que implica el uso del término para la recopilación rigurosa de datos, se proponen los resultados de una investigación de tipo estadístico sobre la producción editorial de cómics en el territorio español, con especial atención a los cómics traducidos, en los años comprendidos entre 2009 y 2015, que representa la época en la que la crisis ha golpeado con más fuerza a todo el sector financiero español y a la sociedad en su conjunto. Consciente de las distintas variables y enfoques que se podrían haber tenido en cuenta y con el afán de limitar el objeto de estudio, el presente artículo se centra en la evolución de la publicación de cómics en un lapso temporal concreto y significativo para arrojar luz sobre el porcentaje que representan las traducciones respecto del total de publicaciones del medio ${ }^{1}$ y sobre la lengua de partida de dichas traducciones. Se cotejarán datos procedentes de distintas fuentes con el afán de averiguar si difieren. Su finalidad es poner el foco en la naturaleza de las lenguas de los títulos publicados para desvelar las tendencias sobre los procesos editoriales. Por tanto, el trabajo se enmarca en una corriente dentro de los Estudios de Traducción que analiza el contexto editorial como parte del análisis de las traducciones. ${ }^{2}$ Asimismo, acomete la tarea de subrayar la falta de estudios previos de este tipo, junto a las contra-

I De acuerdo con los postulados de Barrero (2015: 155) y Altarriba (2011: 9), se ha evitado el uso del término «género» con el fin de atribuir al cómic un carácter emancipado de otras artes.

2 Los Estudios descriptivos de Traducción (EDT) —en los que destaca el trabajo teórico de Gideon Toury-, se caracterizan por analizar empíricamente las traducciones como productos en su contexto cultural y editorial. dicciones que surgen en los mismos trabajos, y tiene el afán de proporcionar datos editoriales que puedan, por un lado, ser significativos para analizar los gustos y las tendencias del mercado a lo largo de un momento histórico con un fuerte impacto social y cultural y, por otro, ofrecer una base sólida y completa para el desarrollo de nuevas investigaciones sobre la traducción del cómic y la relación entre traducción y procesos editoriales. Por ende, poner el foco en la traducción dentro del espectro de los estudios acerca del consumo de cómics en el territorio español será de utilidad para situar en el marco del debate actual el papel que desempeñan las lenguas de los textos de origen bajo el paraguas de los mecanismos editoriales en España.

El 5 de diciembre de 2007, justo dos años antes del marco temporal de este trabajo, la UNESCO planificó en París un encuentro que involucró tanto a editores como a traductores bajo el título «Medir los flujos de traducción: ¿con qué finalidad?». Como afirma Fouces González (2011: 187), «sus premisas son de gran importancia para nuestro trabajo [de investigación]», puesto que ponen de manifiesto el papel de la traducción en el contexto de la globalización y del mercado ya que, gracias a ella, «el intercambio de contenidos editoriales ha experimentado un crecimiento $\sin$ precedentes» (UNESCO, 2007: en línea). Sin embargo, en la mesa redonda también se destaca que «este intercambio es a menudo desigual, y su volumen difícil de cuantificar. La observación de estos flujos es una tarea de primer orden tanto para investigadores como para los actores en materia cultural» (UNESCO, 2007: en línea). Por estas razones, resulta pertinente un estudio de campo que proponga como objeto el cómic bajo la lente de la traducción.

En la primera parte del trabajo se ofrece una fundamentación teórica del estudio, se justifica la elección de las fechas en las que se enmarca 
la investigación y se proporciona información terminológica sobre el cómic y la novela gráfica, poniendo de relieve las contradicciones teóricas y prácticas que derivan del uso de estas palabras y las consecuentes dificultades a la hora de llevar a cabo un estudio sistemático sobre la producción editorial en España.

En la segunda parte del artículo se indaga sobre la metodología empleada a la hora de recopilar la información acerca de la producción global de la edición de cómic y, por ende, de los datos necesarios para el análisis, cotejando fuentes como el Ministerio de Educación, Cultura y Deporte, la plataforma Tebeosfera y Comic Barcelona.

En la última sección se exponen los resultados del estudio de los datos y se presentan, a continuación, las conclusiones.

\section{ESTADO DE LA CUESTIÓN: ¿LA GRAPHIC NOVEL? ¿EL CÓMIC BIOGRÁFICO? ¿LA NOVELA GRÁFICA? UN PROBLEMA TERMINOLÓGICO}

Elaborar un estudio de tipo cuantitativo, como en el caso del presente artículo, presupone definir de forma coherente el lapso de tiempo analizado para alcanzar unas conclusiones significativas. Como se ha expuesto en el apartado anterior, se ha decidido someter a examen la producción editorial de cómic desde una perspectiva de la traducción durante la recesión financiera española y, en concreto, entre 2009 y 2015, siendo el primer año elegido el momento histórico en el cual se materializan las primeras consecuencias e impactos en el mercado ${ }^{3}$ de la gran crisis económica (Tanzi, 2010) y el último año el comienzo de la consolidación de la recuperación económica española.

3 En enero de 2009 Standard \& Poor's es la primera agencia que decide rebajar la calificación crediticia de España y la despoja de la nota máxima, la triple A.
Como se ilustrará a continuación, en el periodo de tiempo analizado, se ha hablado en numerosos medios y plataformas del boom del cómic $y$, en especial modo, de la novela gráfica. En el plano teórico, sin duda, en el territorio nacional, la obra La novela gráfica de Santiago García, publicada por Astiberri en 2010, ha sentado las bases para un verdadero cambio de paradigma hacia la fruición y el estudio del medio. Para García (2010:16), la novela gráfica:

es sólo un término convencional que puede llevar a engaño, pues no hay que entender que con el mismo nos referimos a un cómic con características formales o narrativas de novela literaria, ni tampoco a un formato determinado, sino, sencillamente a un tipo de cómic adulto moderno que reclama lecturas y actitudes distintas del cómic de consumo tradicional. ${ }^{4}$

Por tanto, él mismo como autor pone en cuestión este término y utiliza historieta, tebeo o cómic. Como se estudiará a continuación, se trata de un asunto relevante al ser una consecuencia del por qué existen diferentes datos sobre la producción editorial del cómic en España.

En el plano académico, también han proliferado investigaciones que abordan este tema. En concreto, desde la perspectiva de la traducción, cabría destacar los trabajos de Altenberg y Owen (2015), de Borodo (2015) o, en España, de Las Cuatro de Syldavia (2015). También han surgido iniciativas didácticas enfocadas a la traducción del cómic, como es el caso de las dos ediciones del Curso de Extensión Universitaria en Traducción de Cómic organizado en el marco de titulaciones propias de la Universidad de Málaga en los años 2014 y 2018, cuya finalidad era brindar los conocimientos teóricos y prácticos esenciales para la

4 En 2015 el mismo autor ha publicado en Larousse un nuevo volumen Cómics sensacionales en el que desde otra perspectiva abunda en el tema. 
56 formación y especialización en la traducción de cómic tanto en el ámbito académico como en el profesional. $^{5}$

Asimismo, los medios de comunicación decidieron hacerse eco de este cambio. En 2013, por ejemplo, un titular en la sección de economía del periódico El País rezaba «Cómic y novela gráfica, de la nada al "boom" en cinco años» (Martín, 2013: en línea). En el mismo año la investigadora y periodista Tereixa Constenla ${ }^{6}$ (El País, 2013: en línea) afirmaba que «Marjane Satrapi abrió una vereda con Persépolis». De hecho, el boom de las novelas gráficas se demuestra también a través del éxito, comercial y de crítica, de diversas publicaciones aparecidas en el periodo comprendido desde el premio Pulitzer Maus de Art Spiegelman en 1992 hasta llegar a Persepolis (2000), ${ }^{7}$ a Fun Home $(2006)^{8} \mathrm{o}$ al The Acme Novelty Library $(1998)^{9}$ de Chris Ware. Así pues, la lucha empezada por Will Eisner alrededor de los años 40, cuando en una entrevista para el Baltimore Sun afirmó que «el cómic es una forma artística y literaria legítima» (apud Vélez, 2006: en línea), parece haberse convertido en realidad. En aquella ocasión, el autor intentó defender su tesis enfrentándose a otros artistas contemporáneos como Rude Goldberg (Eisner, 2006: 163):

5 Para profundizar es posible consultar la siguiente página web de la Universidad de Málaga: https://www.titulacionespropias.uma.es/informacion_curso.php?id_curso=6902715 [fecha de consulta: $20 / 05 / 2020$ ]

6 Tereixa Constenla ha sido reconocida con numerosos premios por su trayectoria investigadora y periodística, como el Permio Meridiana, el Premio José de Juan de periodismo, el Premio Nacional de Periodismo Francisco Valdés o el Premio Unicaja de artículos periodísticos.

7 Persepolis. Norma Editorial, Barcelona. Traducción de Albert Agut, 2002.

8 Fun Home. Penguin Random House. Traducción de Rocío de La Maya, 2008.

9 El catálogo de novedades Acme, Penguin Random House. Traducción de Rocío de La Maya, 2009.
$\mathrm{Y}$ los tipos aquellos se rieron en mi cara diciéndome: «¿Qué intentas demostrar, Will? ¿Quién te crees que eres?». Rude Goldberg me dijo que eso que yo decía era una chorrada. «Joder, chico, eres un artista de vodevil. No olvides que esto es un vodevil».

Eisner, tanto desde un enfoque teórico (con Comic and Sequential Art ${ }^{10}$ o Graphic Storytelling and Visual Narrative ${ }^{I l}$ ) como práctico (con obras como A Contract with God, ${ }^{12}$ The Spirit, ${ }^{13}$ etc.), siempre ha defendido la calidad literaria y artística que se halla en el cómic. El concepto de graphic novel empezó a difundirse a partir de 1978 con la publicación de A Contract with God, una trilogía en la cual el autor judío retrata con maestría el crisol de culturas que caracteriza desde siempre la ciudad de Nueva York. Catalogar de graphic novel su obra fue, en principio, un acto de provocación que escondía un mensaje revolucionario y que, de hecho, está cambiando el panorama industrial del mundo del cómic, además de alentar un cambio fundamental en la consideración intelectual del cómic como medio. En la entrevista con Frank Miller, Eisner (ibidem: 44), en relación con su obra, afirma lo siguiente: «Bueno, yo lo intentaba, pero no era consciente de que estaba comenzando una revolución. Sabía que lo que estaba haciendo era algo diferente porque mi intención era que fuese diferente, y porque estaba hablando a un lector

Io Publicado en España bajo el título El cómic y el arte secuencial, Norma Editorial. Traducción de Enrique Sánchez Abulí, 2002.

II Publicado en España bajo el título La narración gráfica, Norma Editorial. Traducción de Enrique Sánchez Abulí, 1998.

I2 Publicado en España bajo el título Conversación con Dios. Norma Editorial, Barcelona. Traducción de Enrique Sánchez Abulí, 1997.

13 Publicado en España bajo el título Spirit. Norma Editorial, Barcelona. Traducción de Enrique Sánchez Abulí, 1987. 
completamente diferente». En este sentido, las obras de autoras y autores independientes demuestran que el mundo de los superhéroes o del mainstream, en general, representan solo uno de los muchos géneros del cómic, y no la única vía. Como afirman Gómez y Rom (2012: 37), «desde la perspectiva culturalista se habla a menudo de la novela gráfica como cómic literario» por la expectación que genera por su calidad visual y literaria. Según mi opinión, el reto que se tienen que plantear las novelas gráficas en el siglo xxi no es «moverse hacia adelante, sino crecer hacia fuera», como afirma McCloud (2001: 26). Es evidente que hoy en día el cómic goza de una relativa legitimación cultural en comparación con otros productos literarios. Por tanto, más que encontrar unas razones para justificar el término, ya que algunos autores han llegado a plantearse sustituirlo por lit comics o literary comics (Schwartz: 2010, 10), se debería incluir la historieta de una forma sistemática en otros tipos de estudios y círculos, entre ellos, el académico ${ }^{14}$.

De hecho, el fenómeno graphic novel ha sacudido los cimientos de la industria de la historieta occidental tanto en grandes centros de producción artística del cómic, como Estados Unidos o Francia, como en mercados periféricos (a los que pertenece, por ejemplo, España). Sin embargo, cabe destacar que ha habido y sigue habiendo una cierta resistencia a la aceptación de la etiqueta novela gráfica por parte de los propios autores, que lo consideran «un pomposo eufemismo» (García, 2010:35), utilizado por parte de las editoriales con fines comerciales. Esto ha gene-

${ }^{14}$ Es preciso señalar que se está asistiendo a una integración cada vez más fructífera de los estudios sobre cómic dentro de la Academia, con una proliferación de tesis doctorales sobre el tema, de cursos y posgrados especializados, de premios oficiales (como el Premio Nacional de Cómic desde 2007) o de revistas específicas (como, en territorio español, Cuadernos de Cómic o Panóptica, entre otras). rado, por un lado, numerosos problemas de traducción del término y, por otro, de catalogación de los cómics, algo que dificulta enormemente estudios como el que se propone en este trabajo.

Como sostiene Santiago García (2010: 33), «todavía falta mucho para que la expresión [ $\mathrm{gra}$ phic novel] se consolide, y de momento convive con otros intentos, como "visual novel", "graphic album", "comic novel" o "novel-in-pictures"». Con todo y con eso, se asiste a una creciente aprobación de la fórmula graphic novel, a pesar de existir varias posturas teóricas acerca de su definición. Pepe Gálvez (apud García, 2010: 35), autor y teórico del cómic, por ejemplo, encuentra la diferencia entre cómic tradicional y novela gráfica en aspectos que prescinden del elemento formal: «el gran avance, el gran salto que la historieta como medio de expresión ha dado estos últimos años no se ha producido tanto en el campo del lenguaje, que también, como en el de la ambición expresiva, en la voluntad de abarcar objetivos narrativos más profundos y más complejos». Por el contrario, Manuel Barrero (2009, en línea), investigador académico y director de tebeosfera.com, ${ }^{15}$ afirma que este concepto no deja de ser una etiqueta escogida por las editoriales en función de los vaivenes del mercado y que encierra una «perversión etimológica»: por un lado, puede aportar beneficios a corto plazo bajo una perspectiva comercial pero, por otro, puede perjudicar al medio y a su concepción a largo plazo puesto que «si los libreros sólo valoran los tebeos con formato de libro y los distribuidores sólo les sirven este tipo de productos, los lectores terminarán desestimando otros formatos y la historieta, en sentido general, acabará no accediendo a tebeos que no satisfagan los requeri-

I5 Tebeosfera.com es una revista teórica sobre el cómic y el humor gráfico y, a la vez, el mayor catálogo de cómics publicados en España. 
mientos de un mercado que solo admite historietas impresas en cartoné o rústica». Desde su postura teórica, la novela gráfica se define como «un tebeo que contiene una obra de historieta monográfica con sello de autor». Asimismo, subraya Barrero la desafortunada combinación de los términos novela y gráfica, etimológicamente contrapuestos. Además de presentar su opinión, Barrero (ibidem) distingue seis definiciones de la voz novela gráfica:

I. La graphic novel es un tebeo con forma de libro que contiene una historieta de un único autor creada expresamente para esta edición, que trata temas en profundidad (dirigidos a un público maduro) y desarrolla un relato extenso, sin límites editoriales o de formato impuestos previamente, en el que los personajes crecen en complejidad hasta alcanzar un final cerrado.

2. Es un tebeo parecido a un libro, de cualquier temática o género y dirigido a todos los públicos, sobre todo al juvenil o infantil, que goza de protagonistas fijos. Puede ser de uno o varios autores, pero la obra es inédita hasta su aparición con el previsto formato libro, y su extensión y formato vienen generalmente impuestos y limitados por el editor.

3. Es un libro que contiene historietas, sean estas de carácter cómico, fantástico o melodramático, sean estas extensas o cortas, del gusto del joven o del adulto, que además pueden haber sido publicadas con anterioridad por entregas en revistas o diarios.

4. Es un libro ilustrado que narra una historia.

5. Es un producto editorial identificado como «novela gráfica».

6. Es una buena historieta que podría adoptar forma de libro.
Estas posturas, a pesar de ser antitéticas, se sustentan en el error extendido de querer juzgar apriorísticamente una novela gráfica como un producto de calidad superior. Sin embargo, siguiendo las tesis del historietista británico Eddie Campbell ${ }^{16}$, considero que la novela gráfica es un movimiento, no una forma: se trata, pues, de un fenómeno en evolución. Como afirmó en una entrevista publicada en la revista The Comic Journal, «a failed graphic novel is nowhere near as worth keeping as a good comic book» ${ }^{17}$ (Deppey, 2006: 91).

Es innegable, por otra parte, que estas nuevas perspectivas tanto en la producción como en la percepción del cómic se están traduciendo también en un cambio físico de los espacios de venta. La omnívora versatilidad del capitalismo hace que lo mainstream englobe también la novela gráfica como mercado y la obra de autoras como Bechdel o Ware se enmarca dentro de esta corriente. En los últimos años, se asiste a una metamorfosis de grandes almacenes y de librerías (especializadas y generalistas). Sin ir más lejos, a partir de 2014 la Fnac, ${ }^{18}$ que dispone de un amplio espacio dedicado al mundo del cómic, ha creado una sección especial exclusivamente para las novelas gráficas que, antes, pertenecían a la macrocategoría de «cómic».

Todas las reflexiones y distinciones anteriores se pierden en las plataformas estudiadas. Por todas estas razones, no es posible pronunciarse sobre los datos cuantitativos de la novela gráfica como tal.

${ }^{16}$ Es posible leer la traducción integral al castellano realizada por Emilio Martínez del Manifiesto de la novela gráfica de Eddie Campbell en la página web: http://68revoluciones. $\mathrm{com} /$ ?p=450 [fecha de consulta: 22/05/2020].

17 Una novela gráfica fallida es mucho menos interesante que un buen tebeo.

I8 Lo mismo pasa en la web $<$ fnac.es $>$. 


\section{METODOLOGÍA: ¿EL CÓMIC SIGUE «HACIENDO BOOM»?}

La dificultad y las contradicciones que surgen a la hora de definir qué es una novela gráfica, al margen del debate teórico que, como se ha visto, se ha librado, también tiene consecuencias prácticas en el estudio empírico de los cómics, si bien es verdad que, como ha afirmado Borodo (2015: en línea), además de algunos estudios relevantes sobre la traducción del cómic como el de Kaindl (1999), el de Celotti (2008) o el de Zanettin (2008), «it still remains an under-investigated topic within Translation Studies». El concepto de cómic y de novela gráfica también ha influido en la recopilación de datos editoriales, puesto que las fuentes a menudo no coinciden a la hora de proporcionar datos sobre la producción de cómics en España, debido también a la dificultad a la hora de catalogar las publicaciones. Además, como sostienen Gómez y Rom, «el análisis de la industria del cómic en España sufre de un handicap importantísimo: la falta de datos empíricos (especialmente en cuanto a las tiradas y cifras de venta)» (2012: 61).

En este trabajo se han cotejado y estudiado los resultados expuestos por tres fuentes seleccionadas por su solidez, credibilidad, especialización y homogeneidad a la hora de elaborar publicaciones estadísticas. La base documental utilizada ha sido la del Ministerio de Educación, Cultura y Deporte de España, la plataforma Tebeosfera.com y Comic Barcelona, (antiguamente conocido como «Salón Internacional del Cómic de Barcelona»).

Para poder garantizar el rigor y la confiabilidad de la fuente seleccionada para el análisis, se han considerado ciertas reglas esenciales. En concreto, los autores Bardin (1986: 122), Landry (1998: 354), Mayer y Ouellet (1991: 495) señalan cinco.
I. La exhaustividad: la documentación a analizar tiene que permitir clasificar el conjunto del material recogido. En este caso, las lenguas de origen y el año de publicación de cada título.

2. La representatividad: el corpus debe constituir una parte representativa de todos los datos. En este caso, ha sido crucial garantizar la selección de editoriales con un historial sólido y una presencia en todo el territorio nacional.

3. La homogeneidad: la muestra escogida tiene que ser homogénea, elegida según criterios concretos sin presentar elementos singulares con respecto a estos criterios. Para este estudio, se ha verificado, a través de una comprobación manual de los catálogos, que el listado elaborado por el Comic Barcelona haya incluido todas las publicaciones de cada editorial en examen.

4. La pertinencia: el corpus seleccionado debe estar directamente relacionado con el objetivo del análisis. Se ha comprobado, por ejemplo, que las editoriales involucradas publican de forma sistemática cómics $\mathrm{y}$ novelas gráficas.

5. La univocación: es primordial asegurarse que las categorías en examen tengan el mismo sentido para toda investigación.

Con la finalidad de averiguar la lengua de origen de cada título, se ha empleado la base de datos ofrecida por el portal Tiendascosmic. com, que detalla la lengua y el título original de todos los cómics publicados en España, aunque no recoja el nombre de la traductora o traductor, como es posible ver en el siguiente ejemplo. 

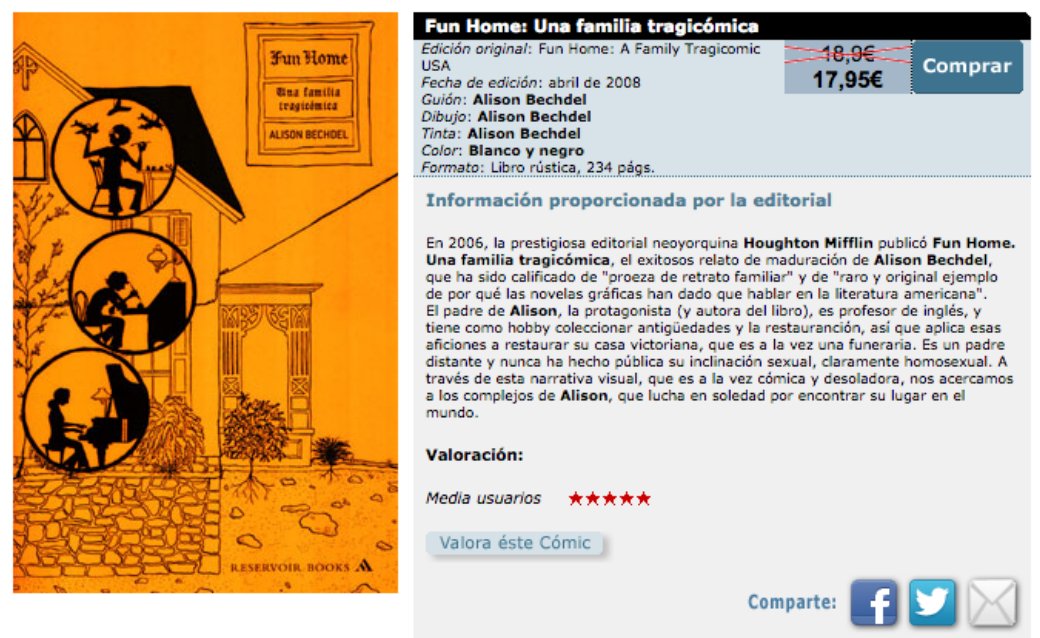

FIGURA I. Ficha de Fun Home elaborada por el portal Tiendascomic.com

\subsection{El Ministerio de Educación, Cultura y Deporte}

La Subdirección General de Promoción del Libro, la Lectura y las Letras Españolas y desde su creación, el Observatorio de la Lectura y el Libro, ambos adscritos a la Dirección General del Libro, Archivos y Bibliotecas del Ministerio de Cultura, elaboran anualmente una publicación estadística en la cual se lleva a cabo un estudio pormenorizado de todas las publicaciones editadas por los agentes españoles que hayan solicitado la asignación de un ISBN, siendo este número el elemento básico para el criterio de selección y de análisis. Por tanto, se incluye también cualquier publicación marginal, siempre y cuando se haya adquirido un número ISBN.

Los datos estadísticos se agrupan por materias y sectores. Es interesante notar que el cómic forma parte del subsector de tiempo libre, «vinculado a temas característicos del tiempo de ocio: Caza y Pesca, Animales Domésticos, Jardinería, Cómics, Economía Doméstica, Juegos y Deportes, Fotografía y Cine, Música, Teatro y Artesanía» (Ministerio de Educación, Cultura y Deporte, 2016: en línea). Así pues, el cómic se encuentra en una categoría compartida con los deportes y las actividades lúdicas y de ocio, lo que implica que los responsables del ISBN consideran que el cómic presenta contenidos más afines a la caza, la pesca, etc. que a las obras de creación literaria. Evidentemente, en este sentido, no existe ninguna diferencia entre el epígrafe de «cómic» y el de «novela gráfica».

En lo que atañe al ámbito de la traducción, el estudio ofrece datos globales sobre el porcentaje de traducciones de cada categoría, sin especificar en ningún momento el país o la lengua de origen. En el caso de los cómics, en el periodo analizado se han extraído los siguientes datos, en los que se marca con una tonalidad de gris más claro el número de cómics autóctonos y en el color más oscuro las traducciones.

Como se deduce de este gráfico, en los años comprendidos entre 2010 y 2014, el porcentaje de cómics traducidos no alcanza las cifras de 


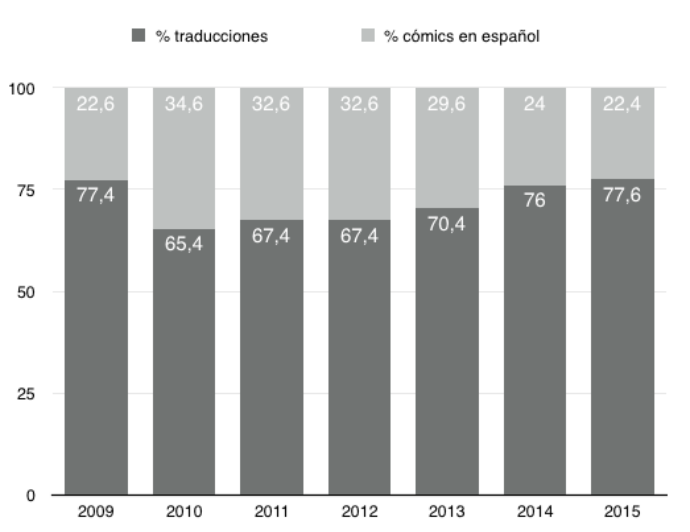

Figura 2. Porcentaje total de cómics traducidos (2009-2015) según el Ministerio de Educación, Cultura y Deporte

2009, probablemente condicionado por la crisis económica en el país y el incremento en los costes que estas implican hasta volver a recuperar gradualmente el número de las traducciones en 2015 con un porcentaje parecido al de 2009.

\subsection{Tebeosfera.com}

La plataforma en línea Tebeosfera.com es un sitio integral sobre historieta, novela, cine, juegos y cultura popular gráfica, gestionado por la Asociación Cultual Tebeosfera, independiente y sin ánimo de lucro, con sede en Sevilla. Es al mismo tiempo un gran catálogo de publicaciones y una revista teórica con secciones divulgativas e informativas anexas. Está integrada por un equipo de especialistas y documentalistas con el objetivo común de estudiar y preservar la historia del cómic en España. El proyecto surgió en 2001 y, hoy en día, es una revista web, pero, sobre todo, un extenso catálogo de publicaciones, artículos, documentos, autores, obras y personajes del cómic, que constituyen el Gran Catálogo de la Historieta con más de 2000 ensayos, artículos, reseñas y recensiones. Entre sus finalidades, es posible destacar la pretensión de generar un corpus de referencia fiable y riguroso. Por estas razones, el equipo de redacción cuenta con el apoyo de agentes externos como coleccionistas, profesionales de la edición y del periodismo y la colaboración de profesores universitarios. Por ende, se ha elegido la plataforma como fuente fiable para llevar a cabo el análisis de esta investigación.

A partir del año 2013 se emite un informe anual con un estudio pormenorizado de las publicaciones impresas catalogadas como tebeos, esto es, cuadernos, revistas y libros. En estos artículos se analizan los cómics desde distintas perspectivas, como el formato (cuadernos, revistas, folletos, libros, antologías, etcétera), el tipo de colección (numerados, únicos, ordenados, convenidos), las lenguas españolas en las obras publicadas (castellano, catalán, euskera, gallego y asturiano) o el reparto por temática y editoriales. Sin embargo, a diferencia de la publicación del Ministerio de Educación, Cultura y Deporte, adquiere especial interés en esta investigación el estudio sobre los tipos de edición y el origen de las obras publicadas en función del país productor. De todas formas, es preciso destacar que no existe en su base de datos ningún informe sobre la situación del cómic desde un punto de vista editorial y de la traducción antes del año 2013. Por tanto, esta fuente solo se ha podido usar de manera parcial para la investigación.

\subsection{Comic Barcelona}

Comic Barcelona es uno de los eventos relacionado con el mundo de la historieta más relevantes en el territorio español a partir de su creación en 1981, que corresponde con el primer éxito del cómic adulto en España a partir de 1977, con la publicación de las primeras revistas que protagonizaron dicho boom. Cada año su programación 
62 incluye talleres de cómic, actividades diseñadas para un público infantil, conferencias, mesas redondas y presentaciones de novedades editoriales. Para este último evento se confecciona un listado con las publicaciones de las editoriales más relevantes en el panorama nacional, dejando fuera los fanzines y las publicaciones marginales que no entran en circuitos de distribución nacionales.

En este caso, no se elabora ningún estudio y todos los datos pertenecientes a esta fuente se han elaborado de manera manual para la investigación.

\section{PRODUCCIÓN GLOBAL DE LA EDICIÓN DE CÓMIC}

Una vez establecidas las bases documentales utilizadas para identificar la presencia de cómics traducidos en el mercado editorial español, es necesario destacar que en este marco se ha elegido el 2009 como punto de partida para realizar la investigación ya que corresponde al primer año en el que se reflejaron las consecuencias de la depresión económica española.

A continuación, se ofrece un gráfico con los datos oficiales proporcionados por el Ministerio de Educación, Cultura y Deporte entre 2009 y 2015.

Si cotejamos las bases de datos ofrecidas por el Ministerio y por Tebeosfera, es posible comprobar que la información no coincide, lo cual pone de manifiesto la dificultad que existe hoy en día a la hora de catalogar las publicaciones del mundo del cómic y de la historieta. Eso se debe a la exclusión de publicaciones con ISSN en los datos del Ministerio. Además, Tebeosfera considera en sus datos publicaciones con cómics, revistas infantiles o incluso productos publicitarios que contienen alguna página de historieta, aunque sean minoritarias. Por el contrario, cada año se han detectado errores $u$ omisiones en estos informes, especialmente en lo que respecta a editoriales muy pequeñas o no especializadas en cómic. Asimismo, en los informes elaborados

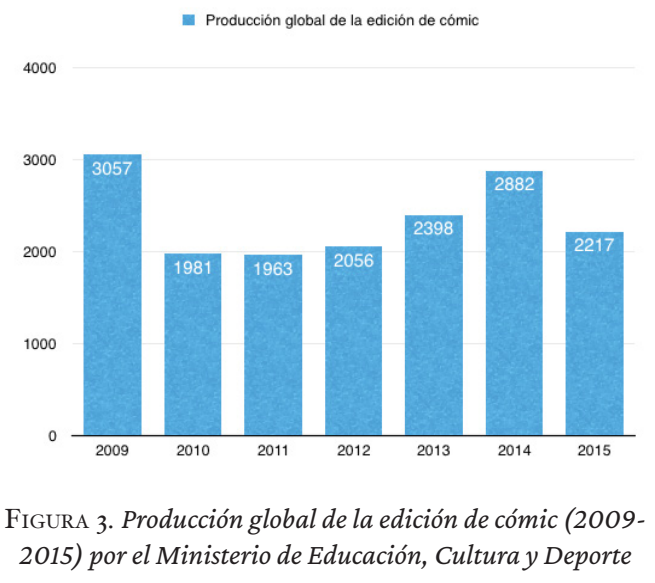

por el Ministerio de Educación, Cultura y Deporte cabe destacar que se incluyen las reimpresiones para el cálculo final de los libros.

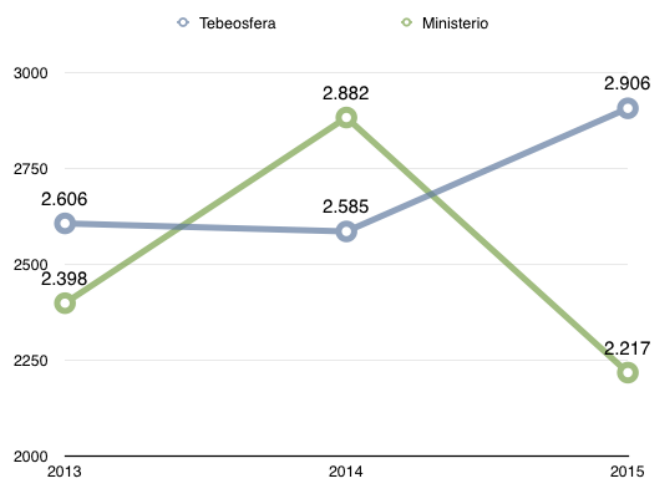

FiguRA 4. Comparación de la producción global de la edición de cómic (2013-2015) entre los informes de Tebeosferay del Ministerio de Educación, Cultura y Deporte

Ante el problema que suponía la contradicción en la recopilación de los cómics, para elaborar esta investigación se ha empleado el corpus proporcionado por Comic Barcelona porque es representativo de las editoriales con más solidez y proyección 
a nivel nacional y puede proporcionar una visión enfocada a las publicaciones con más relevancia en el mercado español. Asimismo, es la única fuente que ofrece el listado completo desglosado de los títulos editados, con lo cual ha sido posible elaborar el estudio de forma autónoma, trabajando singularmente en todas las publicaciones.

\section{OBSERVACIÓN Y RESULTADOS: ¿EL CÓMIC SIGUE «HACIENDO BOOM»? ¿Y SU TRADUCCIÓN?}

Como se ha afirmado anteriormente, para tener una visión objetiva y precisa del papel de la traducción y de la realidad de la novela gráfica en España, se ha llevado a cabo un estudio pormenorizado sobre el estado del cómic a partir del año 2009 hasta el 2015 desde una perspectiva de la traducción, utilizando los listados de novedades editoriales publicados anualmente por Comic Barcelona y cotejándolo con las publicaciones del Ministerio de Educación, Cultura y Deporte, siempre con la finalidad de tener una visión global en comparación con el mundo editorial en su totalidad. Se observa que, a pesar de la crisis económica, el número de cómics publicados en España no ha sufrido grandes variaciones entre 2009 y 2012, es más, en 2013 la producción ha experimentado un fuerte aumento. Sin embargo, en los últimos dos años analizados se asiste a un descenso moderado de la producción de cómics, que los vuelve a colocar en un nivel parecido al 2012, como es posible ver en el gráfico a continuación.

De igual suerte, a pesar de un descenso generalizado en la facturación en las diversas materias, el cómic ha experimentado en los últimos años un aumento de su facturación del 22,5\%, tal y como se recoge en el volumen El sector del libro en España 2011-2013. Observatorio de la Lectura $y$ el Libro publicado por el Ministerio de Educación, Cultura y Deporte (2013: 29).

En cuanto a la traducción, como se estima en otro estudio del Ministerio en el libro Panorámica de la edición española de libros 2012, «las
- Número de cómics publicados 2009-2015

2000

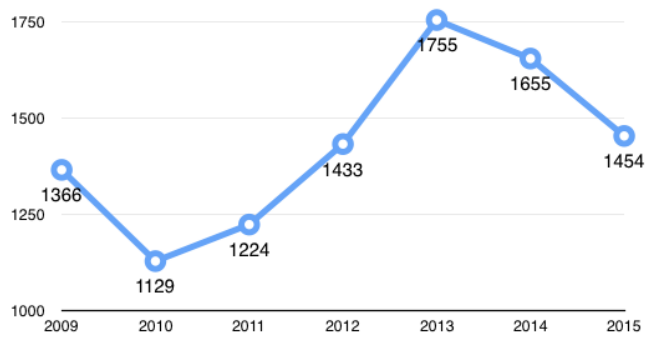

FIgURA 5. Número de cómics publicados entre 2009 y 2015 según Comic Barcelona

traducciones suponen el $22 \%$ del total de la producción editorial española, que en el año 2012 supuso un descenso del 2,1\% del número de títulos traducidos respecto al 2011» (2013: 29). En el mismo estudio de 2015 los datos bajan significativamente: «Las traducciones suponen el 16,2 \% del total de la producción editorial española. En el año 2015 se ha registrado un descenso del 21,1 \% del número de títulos traducidos respecto a 2014» (2015: 27). En el siguiente gráfico se detallan los datos completos.

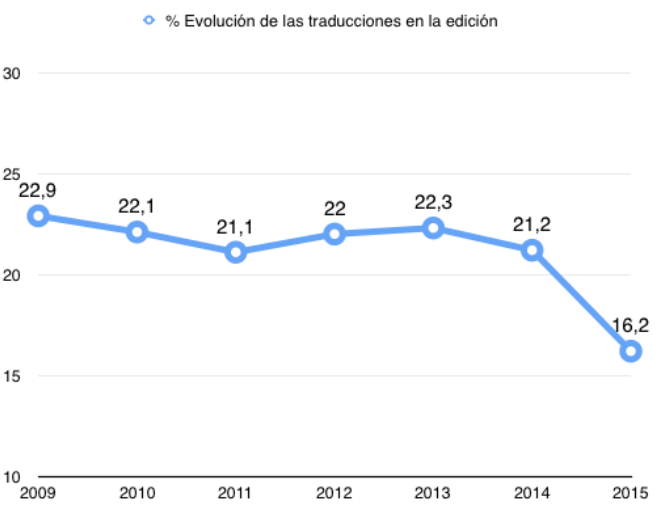

Figura 6. Evolución de las traducciones en la edición (Ministerio de Educación, Cultura y Deporte) 


\begin{tabular}{lccccc}
\hline \multicolumn{5}{c}{ PORCENTAJE DE LENGUAS SOBRE LOS LIBROS TRADUCIDOS } \\
\hline Lenguas & $\mathbf{2 0 0 8}$ & $\mathbf{2 0 0 9}$ & $\mathbf{2 0 1 0}$ & $\mathbf{2 0 1 1}$ & $\mathbf{2 0 1 2}$ \\
\hline Inglés & 49,2 & 47,3 & 48,7 & 47,0 & 51,2 \\
Castellano & 14,4 & 13,9 & 15,3 & 17,2 & 15,9 \\
Francés & 11,7 & 12,2 & 10,9 & 10,5 & 10,3 \\
Italiano & 4,9 & 6,7 & 6,1 & 5,8 & 6,2 \\
Alemán & 5,7 & 6,3 & 5,2 & 6,5 & 5,1 \\
Japonés & 4,2 & 3,8 & 1,4 & 2,2 & 2,6 \\
Catalán & 3,2 & 2,6 & 3,0 & 3,5 & 2,5 \\
Portugués & 0,9 & 1,3 & 0,9 & 1,1 & 0,9 \\
Danés & 0,1 & 0,2 & 0,1 & 0,2 & 0,7 \\
Griego & 0,9 & 0,9 & 2,7 & 0,8 & 0,6 \\
\hline
\end{tabular}

\begin{tabular}{|c|c|c|c|c|c|}
\hline \multicolumn{6}{|c|}{ Porcentaje de lenguas sobre los libros traducidos| } \\
\hline \multirow{2}{*}{ Lenguas } & \multicolumn{3}{|c|}{ Con reimpresiones } & \multicolumn{2}{|c|}{ Sin reimpresiones } \\
\hline & 2012 & 2013 & 2014 & 2014 & 2015 \\
\hline Inglés & 51,2 & 52,3 & 50,2 & 50,1 & 51,7 \\
\hline Castellano & 15,9 & 14,2 & 15,0 & 14,7 & 13,3 \\
\hline Francés & 10,3 & 10,2 & 9,9 & 10,0 & 10,9 \\
\hline Alemán & 5,1 & 6,1 & 5,3 & 4,7 & 5,3 \\
\hline Italiano & 6,2 & 6,2 & 6,0 & 5,0 & 4,9 \\
\hline Japonés & 2,6 & 2,6 & 4,5 & 5,2 & 4,6 \\
\hline Catalán & 2,5 & 2,3 & 2,3 & 2,3 & 3,0 \\
\hline Portugués & 0,9 & 1,0 & 1,2 & 1,2 & 0,9 \\
\hline Ruso & 0,6 & 0,7 & 0,6 & 0,5 & 0,7 \\
\hline Gallego & 0,4 & 0,5 & 0,5 & 0,4 & 0,5 \\
\hline
\end{tabular}

Figura 7. Porcentaje de lenguas sobre los libros traducidos (Ministerio de Educación, Cultura y Deporte)

En concreto, en las tablas anteriores (Ministerio de Educación, Cultura y Deporte, 2013: 30) es posible observar con exactitud el porcentaje de las lenguas sobre los libros traducidos para luego compararlo con los datos del cómic.

Sucesivamente, se cotejarán estos datos con los resultados de mi investigación sobre la producción y la traducción de cómics de 2009 a 2015. La inmensa producción de cómics mainstream norteamericanos hace que el inglés sea el idioma más traducido, seguido por el francés y el japonés, los tres principales mercados productores. Sin embargo, en los últimos dos años se aprecia una leve disminución de los títulos ingleses y japoneses en favor de cómics franceses, italianos y, en su gran mayoría, autóctonos.
A continuación, se facilitan los datos pormenorizados divididos por años y su relación con la publicación global de libros. ${ }^{19}$ En concreto, por cada año estudiado, se proporciona un primer gráfico (de tarta) con el número de cómics y el porcentaje de lenguas de publicación y un segundo gráfico (de barras) en el cual es posible cotejar el porcentaje del número total de libros y el de cómics en relación a la lengua de publicación.

I9 El estudio comparativo no incluye los cómics publicados en 2016 puesto que a la hora de presentar el trabajo de investigación el Ministerio aún no había publicado los datos relativos a este año, con lo cual se ha elaborado exclusivamente el gráfico con el número de cómics y el porcentaje de lenguas de publicación. 


\section{- Añ̃o 2009}
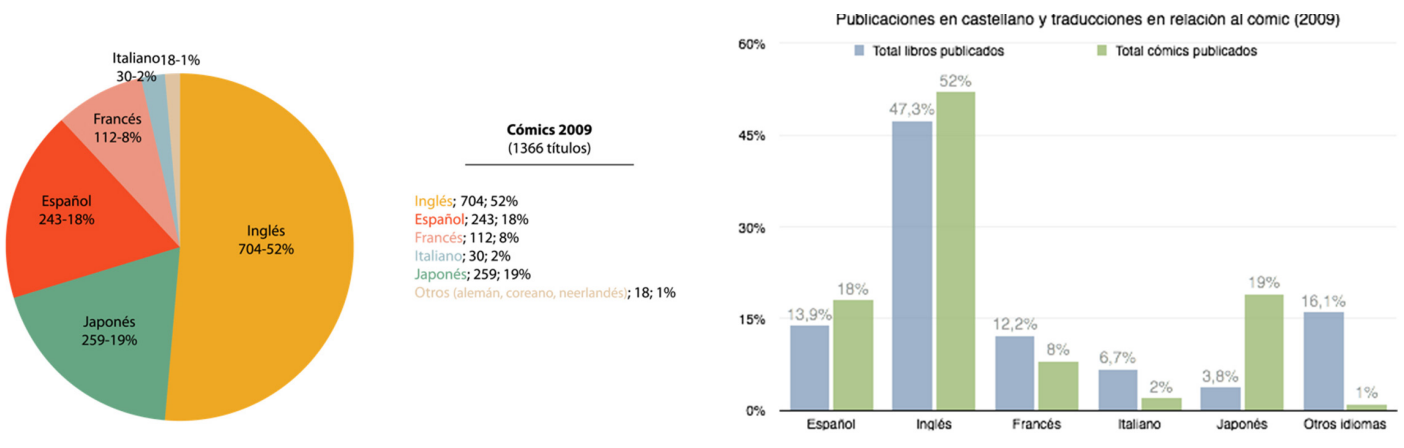

\section{- Añno 2010}
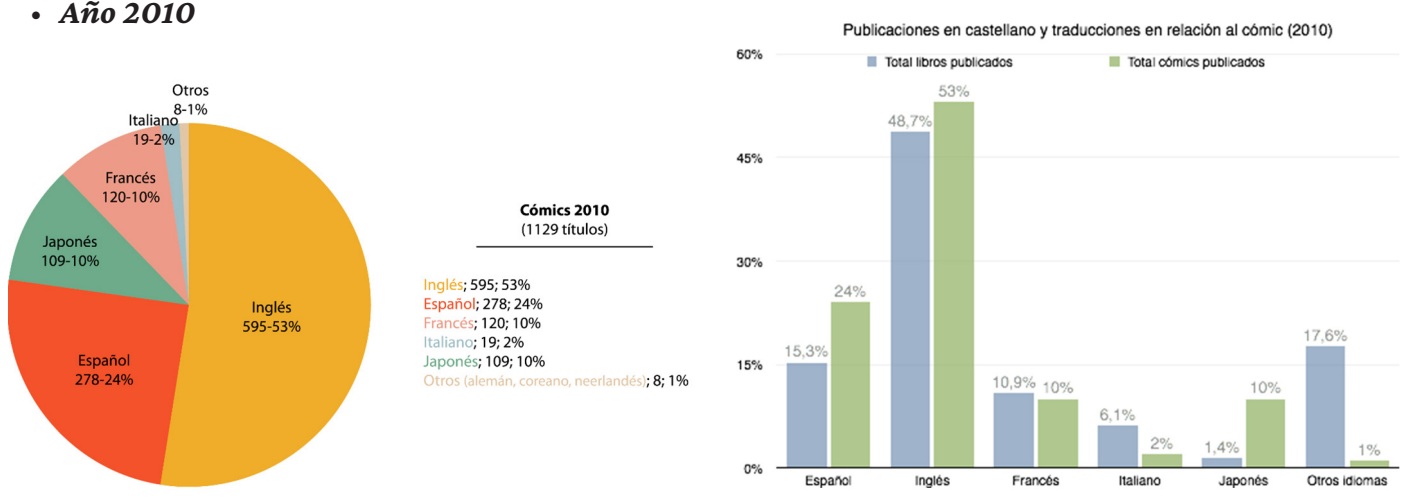

\section{- Año 2011}
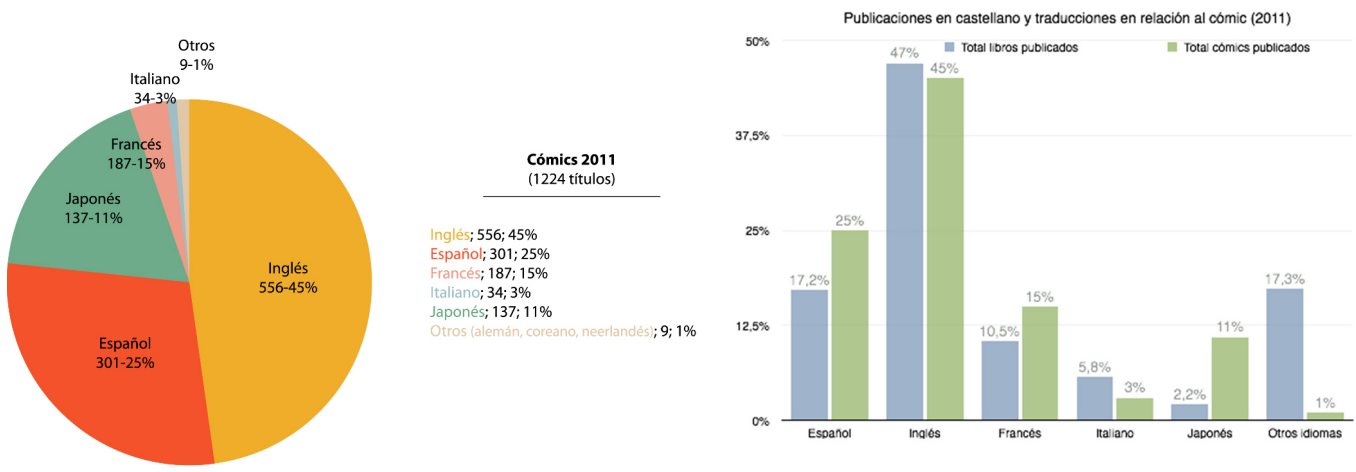
- Añ̄o 2012
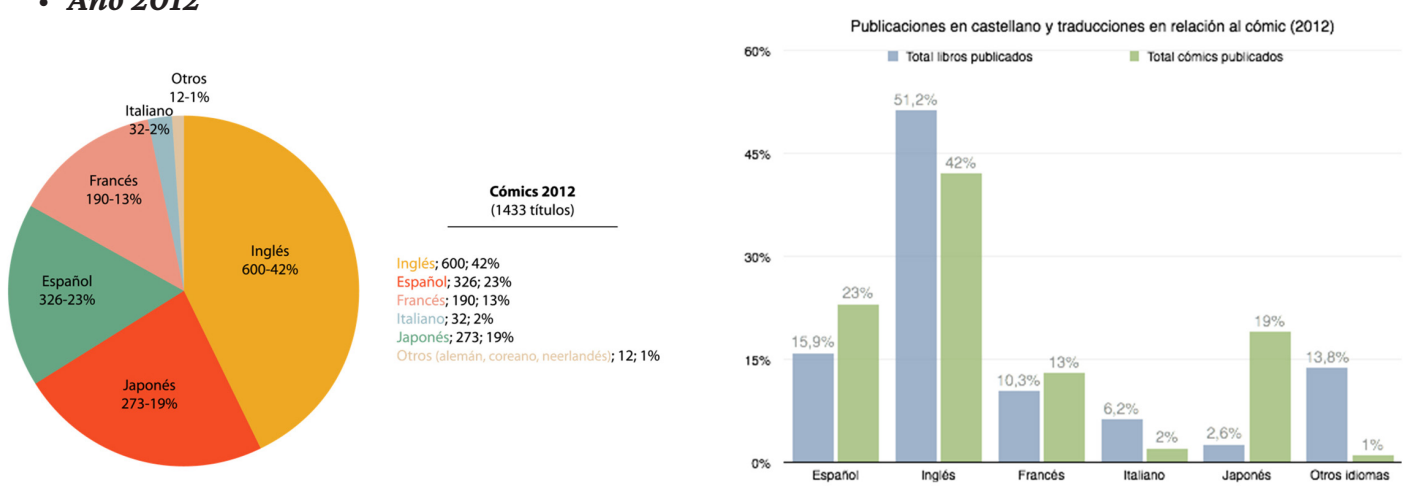

\section{- Añ̃o 2013}
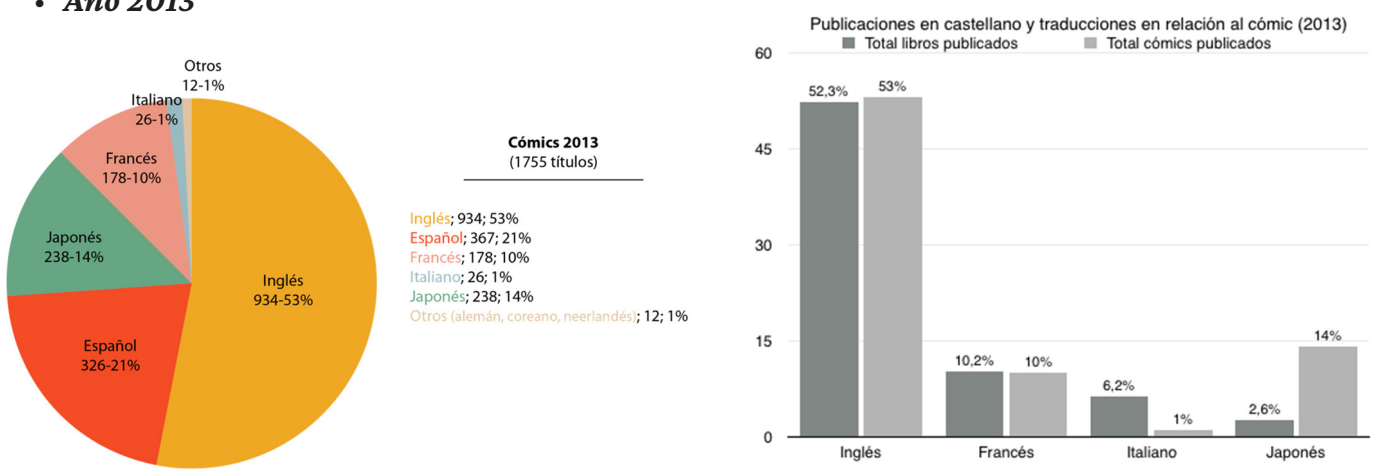

\section{- Añ̃o 2014}
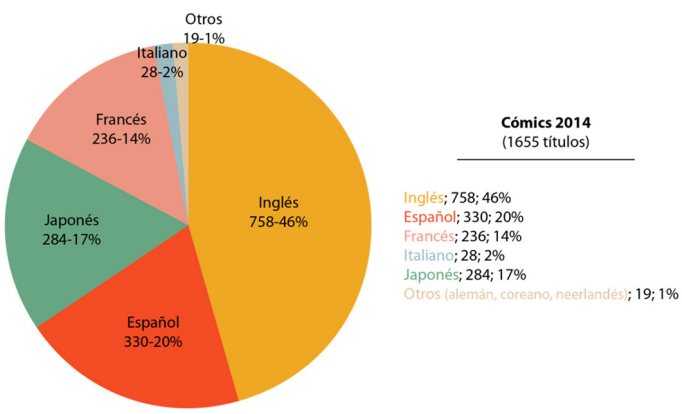

60

Traducciones en general y en relación al cómic (2014)

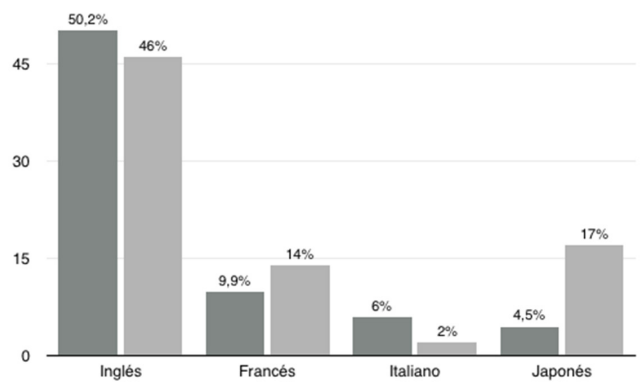


- Año 2015
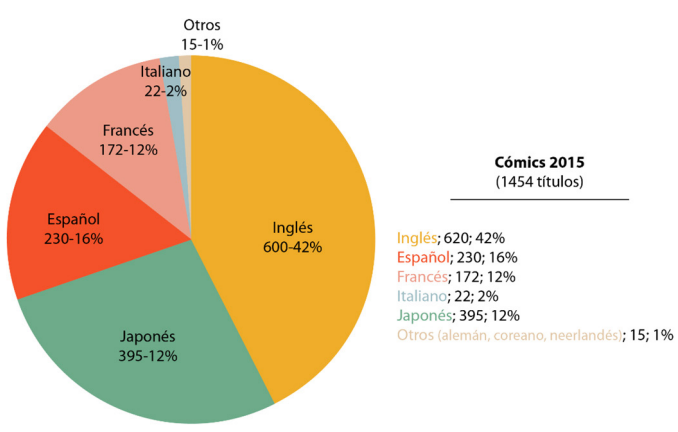

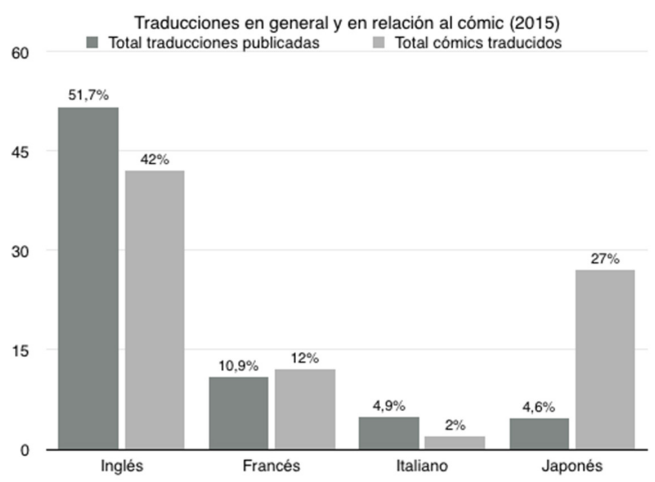

hasta volver a superar en 2015 en dos puntos porcentuales el número de obras traducidas en 2009, tras unos años en que dicho porcentaje había sido más bajo que en 2009. A continuación, se proporciona un gráfico detallado.

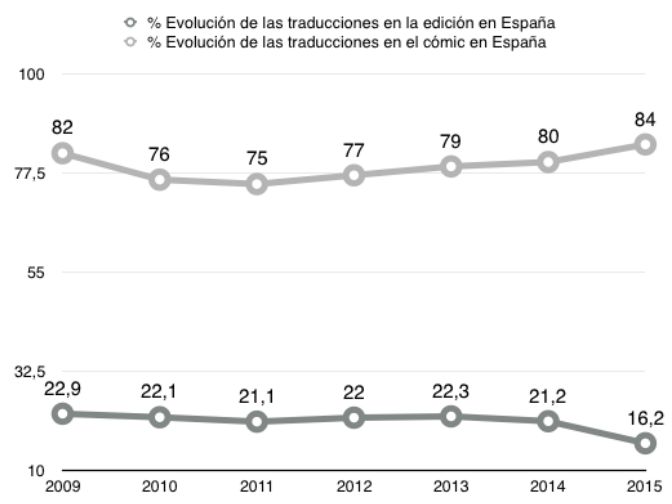

Como es posible observar en el gráfico, el mercado del libro en general ha sufrido unas oscilaciones parecidas, aunque mucho más leves, entre 2010 y 2011 (-1,8\%), actuando de manera disconforme en los últimos dos años analizados, con una fuerte bajada de 6,7 puntos porcentuales con respecto al 2009: se ha pasado de un 22,9\% de obras traducidas al castellano en 2009 hasta ba-
Tras analizar los resultados del estudio, es posible comprobar las tendencias generales de las publicaciones en España en su conjunto y, en concreto, del cómic en relación a la lengua de publicación y traducción entre 2009 y 2015.

Una primera consideración general sustantiva es que la producción de cómic ha ido aumentando durante los años de la recesión económica española, a pesar de experimentar un leve descenso en los últimos años, pero por encima de la producción en 2009, manteniéndose ligeramente por debajo solo en 2010 y 2011. Por tanto, es posible afirmar que «el cómic sigue haciendo boom»y, en concreto, de eso se está beneficiando el mundo de la traducción.

De hecho, en segundo lugar, si comparamos el porcentaje de traducciones totales de la producción editorial española con el del mercado del cómic en el periodo que abarca 2009 y 2015, se deduce que el porcentaje de cómics traducidos supera la media nacional en más de un $53 \%$, colocándose entre un $75 \%$ y un $84 \%$. Se aprecia un descenso en el porcentaje de cómics traducidos durante los años más duros de la recesión económica española (-7\%), con un consecuente aumento de las obras publicadas en castellano,

\section{7}


jar a 16,2 \% en 2015. Por tanto, estos datos arrojan luz sobre la buena salud del mercado de cómics español con respecto al mundo de la traducción, siendo un campo extremadamente más receptivo a la introducción de textos extranjeros con respecto al panorama editorial en su conjunto.

Una tercera conclusión relevante es que las traducciones reflejan el predominio de las tres tradiciones históricas del cómic: la anglosajona, la francobelga y la japonesa. El inglés se coloca como la lengua más traducida, aunque en los últimos años se observa una constante bajada significativa de cómics de tradición anglosajona, hasta llegar a un $-10 \%$ en 2015 . Solo en 2010 y en 2013 el porcentaje se vuelve a colocar en el $53 \%$ :

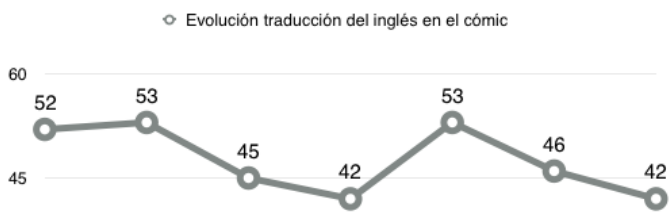

30

15

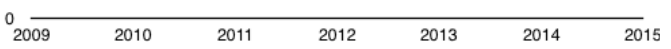

El japonés, la segunda lengua más traducida, ha sido la que más ha sufrido las consecuencias directas de la bajada de traducciones en los años más duros de la crisis, eso es, entre 2010 y 2011, puesto que ha llegado a perder hasta 9 puntos porcentuales. Sin embargo, en los últimos años es la lengua que, con diferencia, ha demostrado un crecimiento sustantivo, llegando en 2015 a un $27 \%$ (un $8 \%$ más con respecto a 2009).

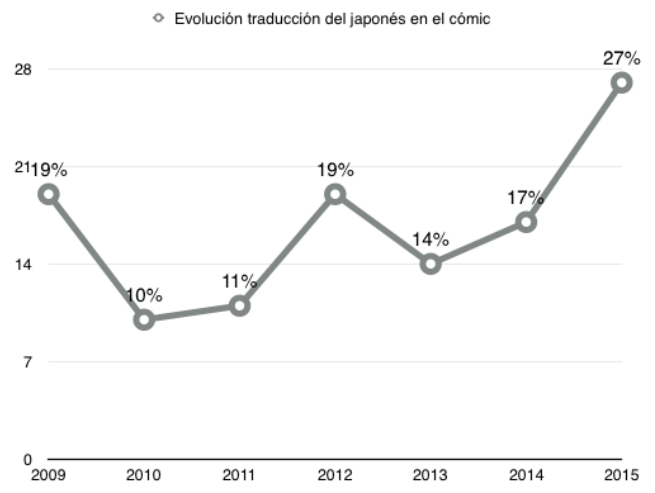

Por su parte, las publicaciones en francés han experimentado una tendencia irregular $y$, en parte, opuesta al inglés: si, por un lado, a lo largo de los seis años analizados, el porcentaje de textos en francés ha subido y bajado en dos ocasiones, por otro, gracias al éxito de las novelas gráficas, se ha observado una subida de entre cuatro y seis puntos en los últimos dos años, rozando los siete en 2011. Es preciso destacar que, a diferencia del inglés y del japonés, que durante el 2010 y el 2011 han experimentado un descenso significativo, los textos traducidos del francés han subido hasta un $7 \%$, doblando casi la cantidad de libros con respecto al 2009:

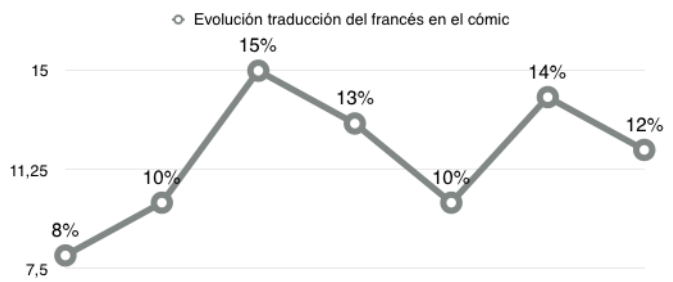

3,75

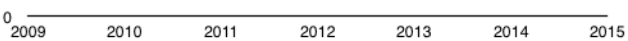


Finalmente, en cuanto a los otros idiomas/literaturas, como el italiano o el alemán, se asiste a un comportamiento homogéneo en el número de cómics y novelas gráficas traducidas entre 2009 y $2015 .^{20}$

\section{REFERENCIAS BIBLIOGRÁFICAS}

Altarriba, A. (2011): «Introducción sobre el origen, evolución, límites y otros debates teóricos en torno a la historieta», Arbor, 187, <http://arbor.revistas. csic.es/index.php/arbor/article/view/1369/1378> [consulta: 14-VIII-2020]

Altenberg, T. y R. J. Owen (2015): «Comic and Translation», en T. Altenberg (ed.), New Readings, vol. 15, ISSN: 13597485, <http://ojs.cf.ac.uk/index.php/ newreadings/issue/view/18/showToc> [consulta: 16-V.2020].

BARDIN, L. (1986): El análisis de contenido, Madrid: Akal.

Barrero, M. (2005): «La novela gráfica. Perversión genérica de una etiqueta editorial», Literaturas, 10, <http://literaturas.com/v010/sec0712/suplemento/Articulo8diciembre.html> [consulta: 22-V2020]

Barrero, M. (2015): Diccionario terminológico de la historieta, Sevilla: ACyT Ediciones.

Borodo, M. (2015): «Multimodality, translation and comics», Perspectives: Studies in Translatology, vol. 23, No 1, 22-41. DOI: <http://dx.doi.org/10.1080/09 07676X.2013.876057> [consulta: 16-V-2020]

Celotti, N. (2008): «The Translator of Comics as a Semiotic Investigator», en F. Zanetrin (ed.), Comics in Translation, Manchester: St Jerome Publishing, 33-49.

Constenla, T. (2013): «Cómic-protesta oriental», El País, 9 de julio, <http://elpais.com/articulo/socie-

\footnotetext{
${ }^{20}$ No quisiera concluir esta investigación sin mencionar la importancia cardinal de incluir en estudios posteriores de esta naturaleza otras variables como las editoriales y un sector fundamental del proceso editorial: el público lector y consumidor. De hecho, es posible ampliar y profundizar este trabajo obteniendo datos sobre la novela gráfica como tal o analizando cómo las etiquetas del estado de la cuestión se reflejan en los catálogos de la editorial.
}

dad/Expedientada/clinica/curar/homosexualidad/elpepisoc/20100615elpepisoc_8/Tes> [consulta: $19-$ IV-2020]

Deppey, D. (2006): «The Eddie Campbell Interview», The Comics Journal, 273, 66-114.

Martín, P. (2013): «Cómic y novela gráfica, de la nada al "boom" en cinco años», El País digital, 8 de junio, <http://economia.elpais.com/econo$\mathrm{mia} / 2013 / 06 / 08 /$ agencias/1370694496_344976. html> [fecha de consulta: 18-V-2020]

EISNER, W. (2006): Eisner/Miller: Entrevista modernada por Charles Brownstein, trad. Raúl Sastre, Barcelona: Norma Editorial.

Fouces GonzÁlez, C. G. (2011): «Mapas de traducción en Europa. La ficción narrativa comercial en Italia y España», TRANS, 15, 117-130.

GARCíA, S. (2010): La novela gráfica, Bilbao: Astiberri.

GARCíA, S. (2015): Cómics sensacionales, Barcelona: Larousse.

Gómez Salamanca, D. y J. Rom Rodríguez (2012): «La novel-la gràfica: un canvi d'horitzó en la indústria del còmic», Ítaca. Revista de Filologia, 3, ISSN 21725500, 35-65.

KaIndL, K. (1999): «Thump. Whizz, Poom: A Framework for the Study of Comics under Translation», Target, 11:2, Amsterdam: John Benjamins, 263-288.

LANDRY, R. (1998): «L ' analyse de contenu», en B. GAUTHIER (ed.), Recherche sociale. De la problemátique à la collecte des données, Sillery: Presses de l'Université du Québec, 329-356.

Las Cuatro De Syldavia (2015): «Ceci n'est pas un tebeo», Vasos Comunicantes, 46, 55-73.

MAYER, R. y F. OuelLEt (1991): Méthodologie de recherche pour les intervenants sociaux, Boucherville: Gaëtan Morin Éditeur.

McCloud, S. (2001): La revolución de los cómics, trad. Estudio Fénix, Barcelona: Norma Editorial.

Ministerio de Educación, Cultura y Deporte (2013): El sector del libro en España 2011-2013, Madrid: Ministerio de Educación, Ciencia y Deporte, <http:// mcu.es/libro/docs/MC/Observatorio/pdf/Sector_ Libro_2011_13_sept13.pdf> [consulta: 20-V-2020]

Ministerio de Educación, Cultura y Deporte (2016): Panorámica de la edición española de libros 2015. Análisis sectorial del libro, Madrid: Ministerio de Educación, 
70 Ciencia y Deporte, <http://sede.educacion.gob.es/ publiventa/panoramica-de-la-edicion-espanolade-libros-2015-analisis-sectorial-del-libro/librosy-lectura/20784C> [consulta: 27-V-2020]

Schwartz, B. (2010): «Introduction», en B. Schwartz (ed.), Best American Comics Criticism, Seattle: Fantagraphic Books.

TANZI, V. (2010): La crisis financiera y económica de 2008-2009: efectos fiscales y monetarios, <https:// asip.org.ar/la-crisis-financiera-y-economicade-2008-2009-efectos-fiscales-y-monetarios/> [consulta: $20-\mathrm{V}-2020]$

Unesco (2007): Medir los flujos de traducción: ¿con qué finalidad?, <http://portal.unesco.org/culture/en/ev.php-URL_ID $=7810 \&$ URL_DO $=$ DO_ $_{-}$ TOPIC\&URL_SECTION=201.html> [consulta: 25$\mathrm{V}-2020]$

Vélez, A. (2006): «El espíritu de Will Esiner», 68 revoluciones, <http://68revoluciones.com/?p=432> [consulta: 13-V-2020]

Zanettin, F. (2008): Comics in translation, Manchester: St. Jerome Publishing. 